Pacific Journal of Mathematic 


\section{A DECOMPOSITION OF COMPLETE BOOLEAN ALGEBRAS}

\section{S. ARgYROS}

Denoting by $|B|$ the cardinality of a Boolean algebra $B$ and by $S(B)$ the least cardinal $\kappa$ such that every family of mutually disjoint elements of $B$ has cardinality less than $\kappa$, we prove that: if $B$ is a complete Boolean algebras, then there is a finite family $B_{1}, \cdots, B_{n}$ of complete Boolean algebra, such that $B$ is isomorphic to the product $B_{1} \times \cdots \times B_{n}$, and $\left|B_{i}\right| S^{\left(B_{i}\right)}=\left|B_{i}\right|$ for $i=1,2, \cdots, n$.

Pierce has proved in [2] that $|B|^{\omega}=|B|$ for every infinite complete Boolean algebra. Comfort and Hager in [1] extended this resut to the class of infinite countably complete Boolean algebras, and also provided some counterexamples of complete Boolean algebras, such that $|B|^{\lambda}>|B|$ for some cardinal $\lambda<S(B)$, the Souslin number of (the Stone space of) $B$. In the present paper we examine the structure of the class of infinite complete Boolean algebras for which the above inequality holds.

The organization of the paper is as follows.

In $\S 1$ we establish some simple relations on cardinal arithmetic.

In $\S 2$ we give the proof of the main result in topological form (via Stone's duality). Also we give some sufficiency conditions for the equality $|B|^{S(E)}=|B|$ and the extension of its validity to the class of $\kappa$-disconnected compact Hausdorff spaces.

The author wishes to thank the anonymous referee for his very constructive suggestions.

o. Preliminaries. The symbols $\xi, \sigma$ denote ordinals and the symbols $\alpha, \beta, \cdots$ denote cardinals.

For a cardinal $\alpha$ we denote by $c f(\alpha)$ the least cardinal $\beta$ such that $\alpha$ is a sum of $\beta$ many cardinals less than $\alpha$. The cardinality of a set $A$ is denoted by $|A|$.

For $\alpha, \kappa$ we set $\alpha^{\kappa}=\sum_{i<\kappa} \alpha^{\lambda}$.

A cardinal $\alpha$ is strongly $\kappa$-inaccessible if for each $\beta<\alpha$ and $\lambda<\kappa$ we have $\beta^{\lambda}\langle\alpha$, if in addition $\alpha>\kappa$ then we write $\alpha \gg \kappa$.

We consider only infinite $T_{2}$-topological spaces. Let $X$ be compact topological space. The weight of $X$, denoted by $w(X)$, is the least cardinality of a base for the topology of $X$. The Souslin number of $X$ denoted by $S(X)$, is the least cardinal $\kappa$ such that there is no set of open mutually disjoint subsets of $X$ of cardinality $\kappa$. 
1. Roots of cardinals and their properties.

Definitions 1.1. Let $\alpha, \kappa$ be cardinals. We set

$$
\begin{aligned}
& \sqrt[\kappa]{\alpha}=\min \left\{\beta: \beta^{\kappa} \geqq \alpha\right\} \\
& \sqrt[\kappa]{\alpha}=\min \{\sqrt[\lambda]{\alpha}: \mu<\kappa\} .
\end{aligned}
$$

It is clear that

$$
\begin{aligned}
& \text { if } \lambda<\kappa, \text { then } 2 \leqq \sqrt[\kappa]{\alpha} \leqq \sqrt[\lambda]{\alpha} \leqq \alpha, \text { also } \\
& \sqrt[\kappa]{\alpha}=\min \left\{\beta \text { : there is } \lambda<\kappa \text { with } \beta^{\lambda} \geqq \alpha\right\} .
\end{aligned}
$$

REMARK 1.2. If $\sqrt[\kappa]{\alpha} \leqq \beta \leqq \alpha$ then $\sqrt[\kappa]{\alpha}=\sqrt[\kappa]{\beta}$. (Indeed, it is clear that $\sqrt[k]{\beta} \leqq \sqrt[\kappa]{\alpha}$; if $\sqrt[k]{\beta}<\sqrt[\kappa]{\alpha}$ then

$$
\sqrt[\kappa]{\alpha} \leqq(\sqrt[\kappa]{\beta})^{\kappa}<\alpha
$$

and hence

$$
(\sqrt[k]{\alpha})^{\kappa} \leqq(\sqrt[k]{\beta})^{\kappa \cdot k}=(\sqrt[k]{\beta})^{\kappa}<\alpha
$$

a contradiction.)

Proposition 1.3. Let $\alpha, \kappa$ be inflnite cardinals and suppose that

$$
2<\sqrt[\kappa]{\alpha}<\alpha
$$

Then, setting $\beta=\sqrt[\kappa]{\alpha}$ we have

(i) $\quad c f(\beta) \leqq \kappa$ and $c f(\beta)<\beta$

(ii) $\beta^{\kappa}=\beta^{c f(\beta)}$.

Proof. Since $\sqrt[\kappa]{\alpha}>2$ we have that $\beta>\kappa$. We prove that $c f(\beta) \leqq \kappa$.

Indeed, suppose that $c f(\beta)>\kappa$. Let $\gamma$ be a regular cardinal such that $\sqrt[k]{\alpha}<\gamma \leqq \alpha$. By Remark 1.2 we have $\sqrt[k]{\gamma}=\beta$. Since $\beta^{\kappa} \geqq \gamma$ it follows that there is one-to-one function

$$
f: \gamma \longrightarrow \beta^{\kappa} .
$$

Define $\phi: \gamma \rightarrow \beta$ by

$$
\phi(\xi)=\sup (f(\xi))(\kappa) .
$$

Since $c f(\beta)>\kappa, \phi$ is well-defined; since $\gamma$ is regular and $\beta<\gamma$ it follows that there is $n_{0}<\beta$ and $A \subset \gamma$ with $|A|=\gamma$ such that 


$$
\phi(\xi)=n_{0} \text { for all } \xi \in A ;
$$

hence

$$
f \mid A: A \longrightarrow n_{0}^{\kappa} \quad \text { is one-to-one }
$$

and from this

$$
\begin{aligned}
& \gamma=|A|=\left|n_{0}\right|^{\kappa} \quad \text { and } \\
& \beta>\left|n_{0}\right| \geqq \sqrt[\kappa]{\gamma} \quad \text { a contradiction. }
\end{aligned}
$$

For the proof of (ii) we observe that

$$
\sqrt[\kappa]{\alpha} \gg \kappa^{+}
$$

So if $\left\{\beta_{\sigma}: \sigma<c f(\beta)\right\}$ is a family of cardinals such that

$$
\begin{gathered}
\sum_{\sigma<c f(\beta)} \beta_{\sigma}=\beta \text { then } \\
\beta^{\kappa}=\left(\sum_{\sigma<c f(\beta)} \cdot \beta_{\sigma}\right)^{\kappa}=\left(\prod_{\sigma<c f(\beta)} \beta_{\sigma}\right)^{\kappa}=\prod_{\sigma}\left(\beta_{\sigma}\right)^{\kappa} \\
=\prod_{\sigma<c f(\beta)} \beta=\beta^{c f(\beta)}
\end{gathered}
$$

where $\beta_{\sigma}^{*}<\beta$ by $\left(^{*}\right)$.

Proposition 1.4. Let $\alpha, \kappa$ be cardinals with $2<\sqrt[\kappa]{\alpha}<\alpha$. Setting $\beta=\sqrt[k]{\alpha}$ we have

(i) $c f(\beta)<\kappa, c f(\beta)<\beta$

(ii) $c f(\beta)=\min \{\lambda<\kappa: \sqrt[k]{\alpha}=\sqrt[\lambda]{\alpha}\}$

(iii) $\beta^{c f(\beta)}=\beta^{k}=\sum_{i<k} \cdot \beta^{\lambda}$.

Proof. By Definition 1.1 and using the Proposition 1.3, we immediately prove (i). In order to prove (ii) we observe that for a $\mu<\kappa$ such that $\sqrt[k]{\alpha}=\sqrt[\mu]{\alpha}$, we have $\beta^{\text {cf( }(\beta)}=\beta^{\mu}$ (by Proposition 1.3). On the other hand using Tarski's recursion formula [3] and the fact that $\beta \gg c f(\beta)$ we have

$$
\beta^{\tau}=\beta \quad \text { for every } \tau<c f(\beta) .
$$

So the proof of (ii) is complete.

Using (ii) we have

$$
\beta^{\kappa}=\sum_{\lambda<k} \beta^{\lambda}=\sum_{c f(\beta) \leqq \lambda<k} \cdot \beta^{\lambda} \leqq \kappa \cdot \beta^{c f(\beta)}=\beta^{c f(\beta)}
$$

which proves (iii).

2. It is known that for each complete Boolean algebra $B$ there is an extremally disconnected space $X(B)$ such that $B$ is isomorphic 
to the algebra of open-and-closed (clopen) subsets of $X$ and consequently

$$
\begin{aligned}
& w(X(B))=|B|, \\
& S(B)=S(X(B)) \quad \text { (Stone's duality theorem). }
\end{aligned}
$$

In the sequel, we consider extremally disconnected compact spaces instead of complete Boolean algebras.

LEMMA 2.1. Let $X$ be compact extremally disconnected space and $\left\{U_{i}: i \in I\right\}$ an infinite family of open nonempty mutually disjoint subsets of $X$ with $w\left(U_{i}\right)=\tau_{i}$ for all $i \in I$, then

$$
w\left(\operatorname{cl}\left(\cup U_{i}\right)\right)=\max \left(2^{|I|}, \prod_{i \in I} \tau_{i}\right) .
$$

Proposition 2.2. Let $X$ be a compact extremally disconnected space with $w(X)=\alpha$. We set

$$
\begin{gathered}
\rho=\min \{\kappa: \text { there is } U \subset X \text { clopen with } \\
w(U)=\alpha \text { and } S(U)=\kappa\} .
\end{gathered}
$$

Then

$$
\alpha^{\tau}=\alpha \text { for each } \tau<\rho .
$$

Proof. Suppose that there is a space $X$ for which the proposition is not valid. Then there is $V$ clopen subset of $X$ and $\tau<\rho$ such that

$w(V)=\alpha$ and $\alpha^{\tau}>\alpha . \quad$ By Remark 1.2 we have $\sqrt[\tau]{\alpha}=\sqrt[\tau]{\alpha^{\tau}}$ which implies $\sqrt[\tau]{\alpha^{\tau}}<\alpha^{\tau}$. By Proposition 1.4 we have either

$$
\begin{aligned}
& \sqrt[\rho]{\alpha}=2, \text { or } \\
& c f(\beta)<\rho \text { and } \\
& \beta^{c f(\beta)}=\beta^{\rho}>\alpha \text { where } \beta=\sqrt[\rho]{\alpha} .
\end{aligned}
$$

In the case of $\sqrt[\rho]{\alpha}=2$ there is $\tau<\rho$ such that $2^{\tau}>\alpha$. Choosing a family $\left\{V_{\xi}: \xi<\tau\right\}$ of mutually disjoint clopen subsets of $V$ we have $w\left(\operatorname{cl}\left(\cup V_{\xi}\right)\right) \geqq 2^{\tau}>\alpha$ (by Lemma 2.1), which is a contradiction. Assuming $2<\sqrt[e]{\alpha}$ the following two cases can be considered.

Case 1. There is clopen $U \subset V$ with $w(U)=\alpha$ and for each clopen $T \subset U, w(T) \geqq \beta=\sqrt[e]{\alpha}$.

In this case, there is a family $\left\{T_{\xi}: \xi<c f(\beta)\right\}$ of clopen nonempty mutually disjoint subsets of $U$, since $S(U)=\rho$ and $c f(\beta)<\rho$. 
This implies $\alpha \geqq w\left(\operatorname{cl}\left(\mathrm{U}_{\xi<c f(\beta)} T_{\xi}\right)\right) \geqq \beta^{c f(\beta)}=\beta^{g}>\alpha$ which is a contradiction.

Case 2. For each clopen $U \subset V$ with $w(U)=\alpha$ there is $T \subset U$ clopen with $w(T)<\beta$.

Using Zorn's lemma we choose a maximal farmily $\left\{T_{i}: i \in I\right\}$ of clopen disjoint subsets of $V$ such that $w\left(T_{i}\right)=\tau_{i}<\beta$ for all $i \in I$.

We assert that $\sup \left\{\tau_{i}: i \in I\right\}=\tau=\beta$. Indeed, if $\tau$ is less than $\beta$ then, since $|I|<\rho$ and $\beta$ is strongly $\rho$-inaccessible, we have

$$
\tau^{|I|}<\beta
$$

and

$$
\beta<w(V)=w\left(\operatorname{cl}\left(\cup T_{i}\right)\right)=\prod_{i \in I} \tau_{i} \leqq \tau^{|I|}<\beta
$$

which is a contradiction.

So there is a family $\left\{i_{\xi}: \xi<c f(\beta)\right\}$ such that $\sup \left\{\tau_{i \xi}: \xi<c f(\beta)\right\}=\beta$ and using Proposition 1.4 and Lemma 2.1 we have

$$
w\left(\operatorname{cl}\left(\cup T_{i_{\xi}}\right)\right)=\prod_{\xi<c f(\beta)} \tau_{i_{\xi}}=\beta^{c f(\beta)}>\alpha
$$

a contradiction. The proof is complete.

In the following corollary we give a different proof of the wellknown result of Pierce which is contained in [2].

CoROllary 2.3. Let $X$ be a compact extremally disconnected space and $w(X)=\alpha$. Then $\alpha^{\omega}=\alpha$.

Proof. For each $T_{2}$-space the Souslin number is uncounable; so the cardinal $\rho$ as it is defined in the previous proposition is uncountable, so this proves the desired statement.

THEOREM 2.4. Let $X$ be a compact extremally disconnected space with $w(X)=\alpha$ and $S(X)=\kappa$.

Then either

(i) for each $\lambda<\kappa \alpha^{\lambda}=\alpha$, or

(ii) there are $U_{1}, U_{2}$ disjoint clopen sets such that

$$
\begin{aligned}
& X=U_{1} \cup U_{2}, \\
& w\left(U_{2}\right)<\alpha, \\
& S\left(U_{1}\right)<\kappa, \quad \text { and } \\
& \alpha^{S\left(n_{1}\right)}=\alpha .
\end{aligned}
$$

Proof. Assume that (i) fails. Let $\left\{U_{i}: i \in I\right\}$ be a maximal family of pairwise disjoint clopen subsets of $X$ such that for each 
$i \in I w\left(U_{i}\right)=\alpha, S\left(U_{i}\right)=\rho_{i}$ and for each $\mu<\rho_{i}$ we have $\alpha^{\mu}=\alpha$. It follows from Proposition 2.2 that if we set $V=\bar{U}_{i \in I} \bar{U}_{i}$ then $w(X-V)<\alpha$.

Setting $S(V)=\tau$ we prove now that, for each $\mu<\tau, \alpha^{\mu}=\alpha$.

Let $\rho=\sup \left\{\rho_{i}: i \in I\right\}$ and we assume first that $\rho \leqq|I|$. Then it is obvious that $\tau=|I|^{+}$and since $w\left(U_{i}\right)=w(X)=\alpha$ for each $i \in I$, and $X$ is an extremally disconnected space, we have that $\alpha^{|I|}=\alpha$ (Lemma 2.1).

So it remains to examine the case where $|I|<\rho$. In this case we assert that either

(1) there is $i_{0} \in I$ such that $\rho=\rho_{i_{0}}$,

or

(2) $c f(\rho)<\rho$.

In fact, if we assume that $\rho>\rho_{i}$ for all $i \in I$, then $\rho$ is a limit cardinal; and if $\rho$ is regular then we have that $|I|=\rho$, a contradiction. Now under the assumption that $|I|=\rho$ we have that $\tau=\rho$ if $\rho=\rho_{i_{0}}$ for some $i_{0} \in I$, and that $\tau=\rho^{+}$, otherwise.

If $\tau=\rho=\rho_{i_{0}}$ then the definition of $\rho_{i_{0}}$ implies that $\alpha^{\mu}=\alpha$ for all $\mu<\tau$. In the case where $\tau=\rho^{+}$we choose a family $\left\{\rho_{\sigma}: \sigma<c f(\rho)\right\}$ such that $\rho_{\sigma}<\rho$ and $\sum \rho_{\sigma}=\rho$; since for each $\sigma<c f(\rho)$ there is $i_{\sigma} \in I$ with $\rho_{i_{\sigma}}>\rho_{\sigma}$ it follows that $\alpha^{\rho_{\sigma}}=\alpha$ and so

$$
\alpha^{\rho}=\prod_{\sigma} \alpha^{\rho_{\sigma}}=\prod_{\sigma} \alpha=\alpha^{c f(\rho)}=\alpha .
$$

We set $U_{1}=V$ and $U_{2}=X-V$; it is easy to see that these two clopen sets satisfy condition (ii) of the theorem.

The following result is an immediate consequence of Theorem 2.4.

COROLlaRY 2.5. Let $X$ be an extremally disconnected compact space, for every nonempty clopen subset $U$ of which either.

$$
w(U)=w(X)=\alpha,
$$

or

$$
S(U)=S(X)=\kappa
$$

Then $\alpha^{\kappa}=\alpha$.

REMARK 2.6. Sufficiency conditions for the equality $w(X)^{S(X)}=$ $w(X)$ for extremally disconnected compact spaces $X$ are also given in Theorem 4 of [1]. 
CoROLlaRY 2.7. Let $X$ be a compact extremally disconnected space. Then there is a finite family $\left\{U_{1}, U_{2}, \cdots, U_{n}\right\}$ of clopen nonempty mutually disjoint subsets of $X$ with

(i) $X=\bigcup_{i=1}^{n} U_{i}$

(ii) $w\left(U_{i}\right)=\alpha_{i}, S\left(U_{i}\right)=\rho_{i}$ and $\alpha_{i}^{\rho_{i}}=\alpha_{i}$ for all $i=1, \cdots, n$.

Proof. We inductively choose $U_{1}, U_{2}, \cdots, U_{n}, \cdots$ clopen sets such that

(i) $w\left(U_{1}\right)=\alpha, S\left(U_{1}\right)=\rho_{1}$ and $\alpha^{\rho_{i}}=\alpha$

(ii) assuming that $U_{n}$ have been chosen such that

$$
\begin{aligned}
& w\left(U_{n}\right)=\alpha_{n}, \quad S\left(U_{n}\right)=\rho_{n} \text { and } \\
& \alpha_{n}^{\rho_{i}}=\alpha_{n} \text { for all } n \leqq n_{0},
\end{aligned}
$$

we set $V_{n_{0}}=X-\bigcup_{i=1}^{n_{0}} U_{i}$.

If $V_{n_{0}}=\varnothing$ then we set $U_{n}=U_{n_{0}}$ for all $n>n_{0}$. If $V_{n_{0}} \neq \varnothing$ then setting $w\left(V_{n_{0}}\right)=\alpha_{n+1}$ and $S\left(V_{n_{0}}\right)=\tau_{n_{0}}$ we consider two cases:

$$
\alpha_{n_{0+1}}^{\tau_{n_{0}}}=\alpha_{n_{0+1}} \text {. }
$$

In this case we set $U_{n}=V_{n_{0}}$ for all $n>n_{0}$

$$
\alpha_{n_{0+1}}^{\tau_{n_{0}}}>\alpha_{n_{0+1}} \text {. }
$$

Using Theorem 2.4 we choose $U_{n_{0}+1} \subset V_{n_{0}}$ such that

$$
\begin{aligned}
& w\left(U_{n_{0}+1}\right)=\alpha_{n_{0}+1}, \quad S\left(U_{n_{0}+1}\right)=\rho_{n_{0}+1}, \\
& w\left(V_{n_{0}}-U_{n_{0}+1}\right)<w\left(V_{n_{0}}\right) \text { and } \\
& \alpha_{n_{0}+1}^{\rho_{n_{0}+1}}=\alpha_{n_{0}+1} .
\end{aligned}
$$

Since the cardinals are well-ordered and the family $\left\{\alpha_{n}: n<\omega\right\}$ is decreasing there is $N<\omega$ such that for each $n>N U_{n}=U_{N}$ and $N$ is the least with this property. That means that the family $\left\{U_{1}, \cdots, U_{N}\right\}$ is as desired.

REMARK 2.8. Analogous statements can be proved for compact $\kappa$-disconnected spaces i.e., for spaces which have a basis of clopen sets such that for any collection $\mathscr{U}$ of its elements with cardinality $\kappa$ the set $\mathrm{cl}(\cup \mathscr{C})$ is also open.

In particular, the following results can be proved with the same arguments as Proposition 2.2 and Theorem 2.4.

Proposition 2.2.a. Let $X$ be a $\kappa$-disconnected space with

$$
w(X)=\alpha .
$$


Then either

$$
\alpha^{\kappa}=\alpha,
$$

or there is $U \subset X$ clopen such that

$$
w(U)=\alpha, \quad S(U)=\rho \leqq \kappa \text { and } \alpha^{\rho}=\alpha .
$$

THEOREM 2.4.a. Let $X$ be $\kappa$-disconnected space with $w(X)=\alpha$ and $\alpha^{\kappa}>\alpha$. Then there are $U_{1}, U_{2}$ clopen subsets of $X$ such that $U_{1} \cup U_{2}=X, S\left(U_{1}\right)=\rho, \alpha^{\circ}=\alpha$ and $w\left(U_{2}\right)<\alpha$.

THEOREM 2.7.a. Let $X$ be a compact $\kappa$-disconnected space with $w(X)=\alpha$ and $\alpha^{\kappa}>\alpha$. Then there is a finite family $\left\{U_{1}, U_{2}, \cdots, U_{n}\right\}$ of clopen nonempty mutually disjoint subsets of $X$ with

(i) $X=\bigcup_{i=1}^{n} U_{i}$,

(ii) $w\left(U_{i}\right)=\alpha_{i}, S\left(U_{i}\right)=\rho_{i}, \alpha^{\rho i}=\alpha_{i}$ for $i=1, \cdots, n$ and

(iii) $U_{i}$ is a complete Boolean algebra for each $i=1, \cdots, n-1$.

REMARK 2.9. Proposition 2.2.a implies, also, that if $X$ is an $\omega$-disconnected compact space then $w(X)^{\omega}=w(X)$ which has been proved in [1] (Theorem 2).

REMARK 2.10. Comfort and Hager in [1] constructed a class of $\kappa$-complete Boolean algebras for which $|B|=\alpha, S(B)>\kappa$, and $\alpha^{\kappa}>\alpha$; furthermore, Theorem 6 in [1] implies that, under some additional set-theoretical assumptions, for example G. C. H., it follows that every $\kappa$-complete Boolean algebra with $|B|=\alpha, S(B)>\kappa$, and $\alpha^{\kappa}>\alpha$ is (isomorphic to) a product $A_{1} \times A_{2}$, where $A_{1}, A_{2}$ are Boolean algebras with

(i) $\left|A_{1}\right|=\alpha,\left|A_{2}\right|<\alpha$,

(ii) if $0 \neq b \in A_{1}$, then $|(b)|=\alpha$

(where we set $(b)=\left\{\alpha \in A_{1}: \alpha \leqq b\right\}$ ),

(iii) $S\left(A_{1}\right) \leqq \kappa$, and

(iv) $A_{1}$ is complete.

Theorem 2.4.a implies that if $B$ is a $\kappa$-complete algebra with $S(B)>\kappa$ and $|B|^{\kappa}>|B|$, then without any set-theoretical assumption, $B$ is isomorphic to a product $A_{1} \times A_{2}$, so that conditions (i), (iii), and (iv) are satisfied. (To the best knowledge of the author, condition (ii) may fail in general, although I know of no specific example).

\section{REFERENCES}

1. W. W. Comfort and A. W. Hager, Cardinality of $\kappa$-complete Boolean algebras, Pacific J. Math., 40 (1972), 541-545. 
2. R. S. Pierce, $A$ note on complete Boolean algebras, Proc. Amer. Math. Soc., 9 (1958), 892-869.

3. A. Tarski, Quelques theorems sur les alephs, Fundam. Math. Soc., 7 (1925), 1-14. Received April 6, 1979 and in revised form May 16, 1979.

Athens University

PANEPISTIMIOPOLIS

Athens 621, Greece 



\section{PACIFIC JOURNAL OF MATHEMATICS}

\section{EDITORS}

DONALD BABBITT (Managing Editor)

University of Galifornia

Los Angeles, California 90024

HUGo RossI

University of Utah

Salt Lake City, UT 84112

C. C. MOORE AND ANDREW OGG

University of California

Berkeley, CA 94720

\section{J. DUGUNDJI}

Department of Mathematics University of Southern California Los Angeles, California 90007

R. FINN AND J. MILGRAM Stanford University Stanford, California 94305

ASSOCIATE EDITORS
E. F. BECKENBACH
B. H. NeumanN
F. WoLF
K. YosHIDA

\section{SUPPORTING INSTITUTIONS}

UNIVERSITY OF BRITISH COLUMBIA UNIVERSITY OF SOUTHERN CALIFONIA CALIFORNIA INSTITUTE OF TECHNOLOGY UNIVERSITY OF CALIFORNIA MONTANA STATE UNIVERSITY STANFORD UNIVERSITY UNIVERSITY OF HAWAII UNIVERSITY OF TOKYO UNIVERSITY OF NEVADA, RENO UNIVERSITY OF UTAH NEW MEXICO STATE UNIVERSITY WASHINGTON STATE UNIVERSITY OREGON STATE UNIVERSITY UNIVERSITY OF OREGON UNIVERSITY OF WASHINGTON 


\section{Pacific Journal of Mathematics \\ Vol. 87, No. $1 \quad$ January, 1980}

Spiros Argyros, A decomposition of complete Boolean algebras ..........

Gerald A. Beer, The approximation of upper semicontinuous multifunctions

by step multifunctions . . . . ....................

Ehrhard Behrends and Richard Evans, Multiplicity theory for Boolean

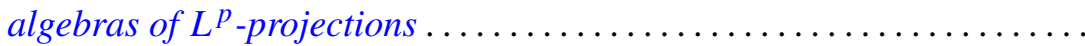

Man-Duen Choi, The full $C^{*}$-algebra of the free group on two

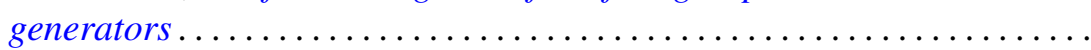

Jen-Chung Chuan, Axioms for closed left ideals in a $C^{*}$-algebra . . . . . . . .

Jo-Ann Deborah Cohen, The strong approximation theorem and locally

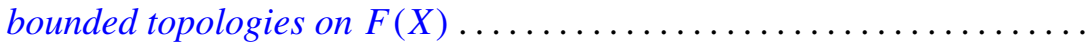

Eugene Harrison Gover and Mark Bernard Ramras, Increasing sequences of Betti numbers............................

Morton Edward Harris, Finite groups having an involution centralizer with

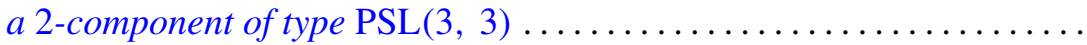

Valéria Botelho de Magalhães Iório, Hopf $C^{*}$-algebras and locally compact

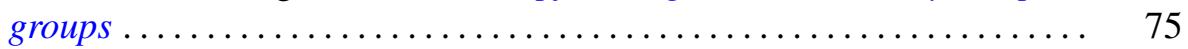

Roy Andrew Johnson, Nearly Borel sets and product measures . . . . . . . . . .

Lowell Edwin Jones, Construction of $Z_{p}$-actions on manifolds . . . . . . . . .

Manuel Lerman and Robert Irving Soare, $d$-simple sets, small sets, and

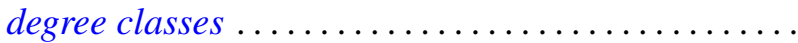

Philip W. McCartney, Neighborly bushes and the Radon-Nikodým property

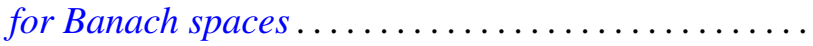

Robert Colman McOwen, Fredholm theory of partial differential equations on complete Riemannian manifolds.

Ernest A. Michael and Carl Preston Pixley, A unified theorem on continuous selections.

Ernest A. Michael, Continuous selections and finite-dimensional sets .

Vassili Nestoridis, Inner functions: noninvariant connected components...

Bun Wong, A maximum principle on Clifford torus and nonexistence of proper holomorphic map from the ball to polydisc.

Steve Wright, Similarity orbits of approximately finite $C^{*}$-algebras . . .

Kenjiro Yanagi, On some fixed point theorems for multivalued

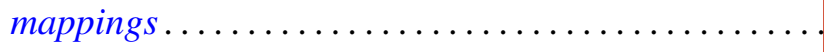

Wieslaw Zelazko, A characterization of LC-nonremovable ideals in commutative Banach algebras 